\title{
Dutch outcome in implantable cardioverter-defibrillator therapy (DO-IT): registry design and baseline characteristics of a prospective observational cohort study to predict appropriate indication for implantable cardioverter-defibrillator
}

\author{
M. van Barreveld ${ }^{1,2}$ • M. G. W. Dijkgraaf ${ }^{3}$ M. Hulleman ${ }^{1}$ L. V. A. Boersma ${ }^{4}$ P. P. H. M. Delnoy • $^{5}$ \\ M. Meine ${ }^{6}$ - A. E. Tuinenburg ${ }^{6}$ D. A. M. J. Theuns ${ }^{7}$ P. H. van der Voort ${ }^{8}$ - G. P. Kimman' ${ }^{9}$ E. Buskens ${ }^{10}$ \\ J. P. G. Tijssen ${ }^{1}$ N. Bruinsma ${ }^{1}$ T. E. Verstraelen ${ }^{1}$ A. H. Zwinderman ${ }^{2}$ P. H. F. M. van Dessel ${ }^{11}$ \\ A. A. M. Wilde ${ }^{1}$ on behalf of the DO-IT investigators \\ Published online: 7 August 2017 \\ (C) The Author(s) 2017. This article is an open access publication.
}

\begin{abstract}
Background Implantable cardioverter-defibrillators (ICDs) are widely used for the prevention of sudden cardiac death. At present, both clinical benefit and cost-effectiveness of ICD therapy in primary prevention patients are topics of discussion, as only a minority of these patients will eventually receive appropriate ICD therapy.

Methods/design The DO-IT Registry is a nationwide prospective cohort with a target enrolment of 1,500 primary prevention ICD patients with reduced left ventricular function in a setting of structural heart disease. The primary outcome measures are death and appropriate ICD therapy for ventricular tachyarrhythmias. Secondary outcome measures are inappropriate ICD therapy, death of any cause, hospitalisation for ICD related complications and for cardiovascular reasons. As of December 2016, data on demographic, clinical, and ICD characteristics of 1,468 patients have been collected. Follow-up will continue up
\end{abstract}

A complete list of investigators in the DO-IT Registry is provided in the online supplementary material.

Electronic supplementary material The online version of this article (doi: 10.1007/s12471-017-1016-x) contains supplementary material, which is available to authorized users.

$\triangle$ M. van Barreveld

m.vanbarreveld@amc.nl

1 Heart Centre, Department of Cardiology, Academic Medical Centre, Amsterdam, The Netherlands

2 Department of Clinical Epidemiology, Biostatistics and Bio-informatics, Academic Medical Centre, Amsterdam, The Netherlands

3 Clinical Research Unit, Academic Medical Centre, Amsterdam, The Netherlands to 24 months after inclusion of the last patient. During follow-up, clinical and ICD data are collected based on the normal follow-up of these patients, assuming ICD interrogations take place every six months and clinical follow-up is once a year. At baseline, the mean age was 66 (standard deviation [SD] 10) years and $27 \%$ were women.

Conclusion The DO-IT Registry represents a real-world nationwide cohort of patients receiving ICDs for primary prevention of sudden cardiac death with reduced left ventricular function in a setting of structural heart disease. The registry investigates the efficacy of the current practice and aims to develop prediction rules to identify subgroups who will not (sufficiently) benefit from ICD implantation and to provide results regarding costs and budget impact of targeted supply of primary preventions ICDs.

4 Department of Cardiology, St. Antonius Hospital, Nieuwegein, The Netherlands

5 Department of Cardiology, Isala Klinieken, Zwolle, The Netherlands

6 Department of Cardiology, Division of Heart and Lungs, University Medical Centre, Utrecht, The Netherlands

7 Department of Cardiology, Erasmus Medical Centre, Rotterdam, The Netherlands

8 Department of Cardiology, Catharina Hospital, Eindhoven, The Netherlands

9 Department of Cardiology, Medical Centre Alkmaar, Alkmaar, The Netherlands

10 Department of Epidemiology, University Medical Centre Groningen, Groningen, The Netherlands

11 Department of Cardiology, Thorax Centre Twente, Medisch Spectrum Twente, Enschede, The Netherlands 
Keywords Defibrillators - Implantable $\cdot$ Heart failure Death · Sudden · Cardiac mortality · Risk assessment . Prospective studies

\section{Background}

Since its introduction in 1980, the implantable cardioverterdefibrillator (ICD) has become a generally accepted therapy for the prevention of sudden cardiac death (SCD) [1]. Its efficacy was demonstrated in both primary and secondary prevention settings [2-8]. Current international guidelines consider primary prevention ICD implantation as a class I indication in patients with New York Heart Association (NYHA) class II or III heart failure with ischaemic or non-ischaemic aetiology and left ventricular dysfunction (left ventricular ejection fraction (LVEF) of $\leq 35 \%$ ). A class I indication is also recommended for patients with an LVEF $\leq 30 \%$ and NYHA I functional capacity $[9,10]$.

At present, both clinical benefit and cost-effectiveness of ICD therapy in patients with a primary prevention indication of SCD are being discussed. During mid-term followup, approximately two thirds of primary prevention ICD patients will never receive appropriate therapy for ventricular arrhythmias [3, 11-13]. They remain at risk of inappropriate shocks and device complications [14-16], which may impair recipients' quality of life [17]. Furthermore, primary prevention randomised control trials (RCTs) were designed and started enrolling patients in the late nineties of the previous century. Both treatment of acute myocardial infarction (primary percutaneous intervention as opposed to thrombolysis) and treatment of heart failure have changed considerably. Today, the question arises whether primary prevention patients will be subject to the same number of

Table 1 Inclusion and Exclusion Criteria

\begin{tabular}{ll}
\hline Inclusion & Criteria \\
1 & ICD implantation for primary prevention of sudden car- \\
diac death & LVEF $\leq 35 \%$ and NYHA $\leq$ III or LVEF $\leq 30 \%$ and \\
2 & NYHA I \\
3 & Life expectancy $\geq 1$ year \\
4 & $\geq 40$ days after myocardial infarction \\
5 & $\geq 90$ days after revascularisation procedure \\
6 & Optimal pharmacological heart failure treatment \\
Exclusion criteria \\
1 & Secondary prevention \\
2 & ICD generator replacement \\
3 & Inability or unwillingness to provide valid informed con- \\
& sent \\
\hline
\end{tabular}

ICD implantable cardioverter-defibrillator, $L V E F$ left ventricular ejection fraction, NYHA New York Heart Association appropriate shocks and will experience a similar relative risk reduction as patients in these 'older' RCTs.

Additionally, there is the ongoing debate whether LVEF values and NYHA functional classes are sufficiently accurate selection criteria for prognostic stratification of SCD risk. Consequently, there is a strong need to improve the current selection criteria for primary prevention ICD treatment in this setting [18-23]. In Europe alone, two prospectively designed cohort studies for improved risk stratification are ongoing [24, 25].

Cost-effectiveness studies show a wide variation in benefit, raising doubt about the efficiency, also regarding costs, of ICD therapy. ICD therapy appears to be only cost-effective in selected patient groups and seems to be related to the number of risk factors present for SCD [22, 26-31]. Risk stratification before ICD implantation is important from both a medical and an economic standpoint.

The DO-IT Registry aims to prospectively register the efficacy of implantation of primary prevention ICDs in order to develop prediction rules to identify subgroups who will not, or not yet, sufficiently or not at all, benefit from ICD implantation for primary prevention of SCD within two years of follow-up. What constitutes 'sufficiently' will be defined in a joint effort with a Dutch convenience sample of ethicists, patient representatives, cardiologists, health care insurers, health care policymakers, and key opinion leaders. Additionally, we aim to assess the costs and budget impact of targeted supply of ICD implantation in primary prevention patients. This article provides an overview of the DOIT Registry design, a cohort description and describes the planned statistical analyses.

\section{Methods}

\section{Design and study population}

Since September 2014, the DO-IT Registry documents the current nationwide practice of ICD implantation in the primary prevention of SCD with reduced left ventricular function in a setting of structural heart disease. We planned to collect data on demographic, clinical, and ICD characteristics of 1,500 consecutively recruited patients in all 28 ICD implanting centres in the Netherlands. Currently, we are collecting follow-up data up to 24 months after inclusion of the last patient. The inclusion and exclusion criteria are shown in Tab. 1. The inclusion criteria are based on current guidelines (e.g. before 2014) for primary prevention ICD implantation $[9,10]$. The LVEF assessment method was at the discretion of the local centre. The registry has been approved by the institutional review boards of all participating hospitals. 


\section{Data collection}

Cardiologists, electrophysiologists, biostatisticians and health economists have jointly defined the relevant data set, in view of existing literature on possible predictors for SCD. Electronic case report forms (e-CRFs) were generated using an open source web-based software platform OpenClinica [32] in compliance with good clinical practice. By randomly generating a unique identification number, the anonymity of the patient is maintained. Data of the patient are collected from medical records and entered into the eCRF. All participating sites access the registry via a personal password protected login procedure and can only access the records of the patients of their own centre. We provided the participating centres with a sequential set of instructions for data entry and offered a training, if needed.

\section{Baseline and follow-up data}

We collected baseline data on demographics, medical history and diagnostics, as well as implantation and device data. We collected clinical and ICD follow-up data, based on the normal follow-up of these patients assuming ICD interrogations take place every six months and clinical follow-up is once a year. Every six months, we register occurrence of ventricular arrhythmias and data regarding ICD therapies. ICD therapy is defined as anti-tachycardia pacing (ATP) and defibrillator shocks. During clinical follow-up, medical history, functional status, cardiovascular hospitalisations and performed procedures and diagnostics of the past year are entered into the registry. In addition, the participating hospitals report adverse events, defined as ICD related complications and deaths of any cause. Events such as death, arrhythmias, cardiovascular hospitalisations, cardiac interventions, diagnostic procedures and laboratory parameters between last follow-up visit and death are documented. Medical documentation around the time of death and ICD interrogations are collected, whenever possible. A more detailed list of the collected parameters is provided in the online supplementary material.

\section{Data and adverse event management}

Verification rules and edit checks have been built into the system to validate completeness, accuracy and consistency of the dataset. Data quality is also maintained by online and on-site monitoring and continuous assessment of data quality during the export of datasets to statistical packages for subsequent analysis. A monitor plan is used which describes whether outcomes and variables need to be monitored entirely. This decision depends on the expected role of the variable in the prediction models, expected frequency of mistakes and the time needed to monitor the variable. The site investigator is required to maintain adequate records to ensure the manner in which the registry is conducted is well documented. The investigator should keep the records on file for a period of time specified by local law for the preservation of hospital documents. If requested, the investigator supplies the monitor with any required data.

A steering committee, represented by cardiologists of the participating hospitals, is responsible for the scientific integrity and the way that the study is conducted and advises the registry coordinating centre, when needed. A clinical event committee, consisting of independent cardiologists, assesses all ICD delivered therapies by analysing stored electrograms. The committee also determines whether deaths are of cardiac origin.

\section{Outcomes}

The primary outcome measures of the DO-IT Registry are death and appropriate ICD therapy for ventricular tachycardia (VT) or ventricular fibrillation. Secondary outcome measures are inappropriate ICD therapy, death of any cause, hospitalisation for ICD related complications and cardiovascular reasons.

\section{Sample size/power}

The registry has a target enrolment of 1,500 patients with a follow-up of at least two years. Based on the latest RCT's and literature $[3,8]$ we expect to observe $150(10 \%)$ patients with appropriate shocks. In combination with 150 fatalities (5\% per year), these are sufficient to develop prediction rules.

\section{Data analysis}

\section{Research aim 1: prediction rules}

The incidence for the outcomes of appropriate ICD therapy and death are illustrated by Kaplan-Meier curves. Cox regression models with baseline characteristics of patients are developed to predict the outcomes of death or appropriate ICD therapy over time, considering the competing risk. The risk models will be developed according to the methods that have been used in the paper on the widely accepted 'MADIT-II risk score' for primary therapy with an ICD [33]. First, we will develop a model with important baseline characteristics, selected from previous publications on ICD mortality risk stratification and appropriate ICD therapy. We will consider age, gender, body mass in- 
Fig. 1 Flowchart of study participants

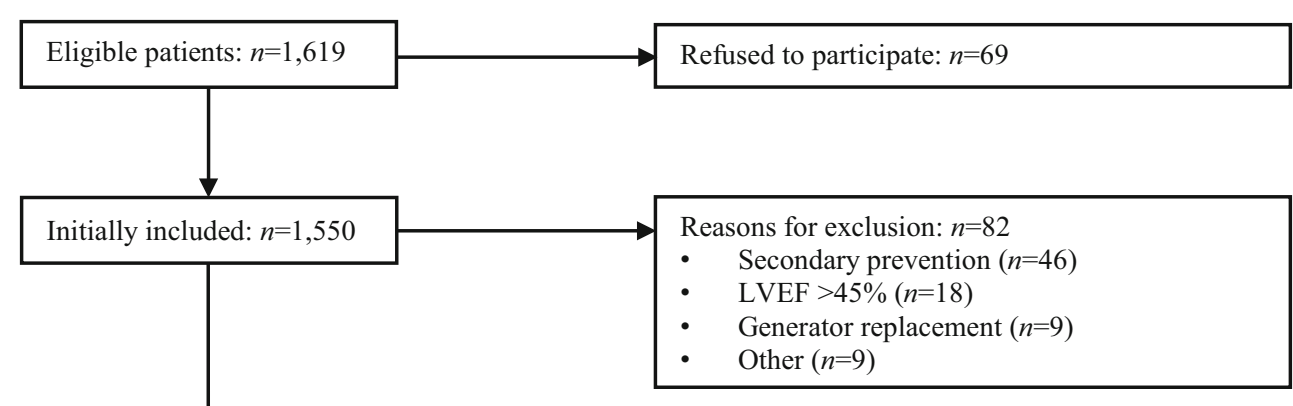

Reasons for exclusion: $n=82$

- $\quad \mathrm{LVEF}>45 \%(n=18)$

- $\operatorname{Other}(n=9)$

ncluded clinically relevant: $n=153$

- $\quad$ LVEF $>30 \%$ and NYHA $1(n=78)$

- $\quad$ PCI $<90$ days $(n=35)$

- $\quad \mathrm{LVEF}>35 \%-45 \%(n=20)$

- $\quad \mathrm{CABG}<90$ days $(n=8)$

- $\quad<40$ days after AMI $(n=5)$

- $\quad$ NYHA IV $(n=3)$

- $\quad$ PCI $<90$ days and $<40$ days after AMI $(n=3)$

- $\quad \mathrm{LVEF}>30 \%$ and $\mathrm{PCI}<90$ days $(n=1)$

$L V E F$ left ventricular ejection fraction, $P C I$ percutaneous coronary intervention, NYHA New York Heart Association,

$A M I$ acute myocardial infarction, $C A B G$ coronary artery bypass grafting.

*Preliminary data as of February 13, 2017. dex, LVEF, NYHA class, prior (<1-year) heart failure hospitalisation, history of atrial fibrillation and non-sustained VT, renal function, diabetes mellitus, smoking history, pulmonary disease and electrocardiographic parameters. Next, we will identify further factors, such as events during follow-up (complications and appropriate shock), that might improve prediction of the primary outcomes in exploratory analyses. The validity of the resulting predictor sets will be assessed by bootstrapping. A total of 200 bootstrap regression analyses will be performed for internal cross validation. The predictive values of the prediction models will be quantified using c-statistics.

\section{Research aim 2: economic evaluation}

A cost-minimisation analysis and a budget impact of targeted supply of ICD implantation will be performed. This economic evaluation will compare targeted supply of ICD implantation based on new prediction rules with current practice as reflected in the DO-IT Registry. Targeted supply, however, is not registered; no ICD implantation is withheld from patients who meet the current guidelines. That is why we are not able to observe what would have happened to these patients had they not received an ICD. To circumvent the missing data, the economic evaluation will be conducted as a modelling study, and assuming that not performing unnecessary ICD implantations does not affect health outcomes, only costs, except for temporal implantation related complications. These assumptions are realistic, but depend on the quality of the prediction rules. That is why we will develop prediction rules which can be applied in a way that will minimise the number of false negative predictions. Further details regarding the cost-minimisation and budget impact analysis are provided in the online supplementary material.

\section{Study population}

Between September 10th, 2014 and May 13th, 2016, 1,619 patients were assessed for eligibility. A flowchart of the study participants is presented in Fig. 1. Sixty-nine patients refused to participate. Data of 1,550 patients were initially entered into the registry. However, 82 patients were excluded because they did not meet the inclusion criteria for various reasons. Although 153 patients did not fully meet the inclusion criteria, we decided that these patients should remain in the registry because they received their ICD in context of primary prevention with a reduced LVEF in a setting of structural heart disease and were assumed to be a clinical relevant subgroup. Therefore, 1,468 patients in total were included in the DO-IT Registry.

\section{Baseline characteristics}

The baseline characteristics are presented in Tab. 2. Overall, 1,468 patients received their first ICD (single-chamber or dual-chamber), subcutaneous ICD (S-ICD) or cardiac resynchronisation therapy defibrillator (CRT-D) for primary 
Table 2 Comparison of patient characteristics of DO-IT Registry (date) with ICD arms of MADIT II and SCD-HeFT Trial

\begin{tabular}{|c|c|c|c|c|}
\hline Patient characteristics & DO-IT $\left(n=1,461^{\mathrm{a}}\right)$ & $\begin{array}{l}\text { DO-IT ( } n=1,308,153 \\
\text { patients excluded) }\end{array}$ & $\begin{array}{l}\text { SCD-HeFT } \\
(n=829)\end{array}$ & $\begin{array}{l}\text { MADIT II } \\
(n=742)\end{array}$ \\
\hline \multicolumn{5}{|l|}{ Age (years) } \\
\hline Mean (SD) & $66(10)$ & $66(10)$ & & $64(10)$ \\
\hline Median (IQR) & $(68)(60-73)$ & $(68)(60-73)$ & $60(52-68)$ & \\
\hline $\operatorname{Men}(\%)$ & 73 & 72 & 77 & 84 \\
\hline \multicolumn{5}{|l|}{ LVEF } \\
\hline Mean (SD) & $26(6)$ & $26(6)$ & & $23(5)$ \\
\hline Median (IQR) & $27(21-30)$ & $26(21-30)$ & $24(19-30)$ & \\
\hline \multicolumn{5}{|l|}{ NYHA } \\
\hline $\mathrm{I}(\%)$ & 14 & 9 & & 35 \\
\hline II $(\%)$ & 61 & 65 & 70 & 35 \\
\hline III $(\%)$ & 22 & 23 & 30 & 25 \\
\hline$(\%)$ & $<1$ & & & 5 \\
\hline Unknown $(\%)$ & 3 & 3 & & \\
\hline Ischaemic cardiomyopathy (\%) & 56 & 54 & 52 & 100 \\
\hline Diabetes $(\%)$ & 27 & 27 & 31 & 33 \\
\hline Hypertension (\%) & 43 & 43 & 55 & 53 \\
\hline Atrial fibrillation (\%) & 30 & 31 & 17 & 9 \\
\hline
\end{tabular}

Data are presented as mean (standard deviation) or median (25th-75th percentiles)

DO-IT Dutch Outcome in ICD therapy,ICD implantable cardioverter-defibrillator, SCD-HeFT Sudden Cardiac Death in Heart Failure Trial, MADIT II Multicentre Automatic Defibrillator Implantation Trial-II, IQR interquartile range, LVEF left ventricular ejection fraction, NYHA New York Heart Association

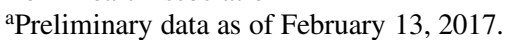

prevention of $\operatorname{SCD}(32 \%, 16 \%, 9 \%$ and $43 \%$, respectively). Seventy-three percent of patients were men and the mean age at implantation was 66 (SD 10) years. The mean LVEF was $26 \%$ and more than half of the patients $(61 \%)$ experienced NYHA class II heart failure symptoms. Most patients, 56\%, had ischaemic heart disease. The remaining $641(44 \%)$ patients were considered non-ischaemic.

\section{Discussion}

In this article, we describe the design, cohort and planned analysis of the DO-IT Registry. This nationwide prospective registry aims to identify patients who will benefit most, or who will benefit the least, from ICD implantation. It further aims to assess the costs and budget impact of targeted supply of primary prevention ICDs. This is one of the largest prospective cohorts of primary prevention ICD patients, with a nationwide participation of all ICD implanting centres in the Netherlands. This research reflects current clinical practice and will provide real-world outcomes in the general population.

There are some differences between the baseline characteristics of the DO-IT patients and the patients included in the largest RCTs, MADIT II [8] and SCD-HEFT [3] (Tab. 2). Overall, DO-IT patients are older, more often female, and have higher LVEF. More patients have NYHA II symptomatic heart failure compared with MADIT II patients.

We enrolled 153 patients who did not fully meet the ACC/AHA/ESC Class I indication for primary prevention ICD implantation. The DO-IT Registry was designed to capture all primary prevention ICD patients. If the reason for ICD implantation was primary prevention for reduced LVEF in the setting of structural heart disease, patients were not excluded from the registry. Prior analyses of other primary prevention ICD registries also show that a proportion of patients do not meet trial inclusion criteria [34, 35]. Further analyses of these patients could clarify the event rate in this group, and might elucidate common risk factors that led to the decision to implant an ICD without a clear Class I indication.

\section{Study limitations}

As expected, the registry has limitations [36, 37]. First, there is no control group with eligible patients who did not receive an ICD. However, the findings can be evaluated within the context of the previous RCTs or other registries. Second, there is a possibility of underreporting or misreporting, because data in registries are often collected more passively and monitored less strictly compared with RCTs. Third, patients should be included consecutively. However, 
the inclusion of patients was not controlled. For instance, sites may have been unable to enrol all eligible patients or simply have forgotten to register one. In addition, physicians may have been more likely to exclude patients with uncertain benefit of ICD implantation, which results in selection bias. However, we built in quality and control checks and online and on-site monitoring to ensure that the data is representative and of adequate quality.

Some recent articles are not discussed in the content of this paper because they were published later than the setup of the DO-IT Registry [38-40]. However, these studies also emphasise the need for improved selection of patients for primary ICD therapy since most patients do not experience benefit.

Although the DO-IT Registry is designed and funded for an initial follow-up duration of two years after inclusion of the last patient, other studies show that patients might benefit from primary prevention ICD therapy with follow-up longer than two years $[11,41]$. However, additional post cohort projections and hypothetical modelling are anticipated. Moreover, the DO-IT Registry intends to extend the observation period if cooperation of the participating hospitals and additional funding can be arranged.

\section{Conclusion}

At present, the indication for primary prevention defibrillators may not be optimal. Many patients do not receive appropriate ICD therapy, but are at risk of inappropriate shocks, device complications, or die without ever needing their ICD. The DO-IT Registry is a large nationwide cohort, consisting of patients receiving ICDs for primary prevention of SCD. Despite the limitations, we expect that the DO-IT Registry will identify subgroups of patients who will not, or not yet, sufficiently benefit from ICD implantation for primary prevention. We also expect that the registry will provide results regarding the costs and budget impact of targeted supply of primary preventions ICDs. Because of real-life data, the findings of this study should be representative and are likely to affect the current risk stratification of SCD.

Funding This work was supported by ZONMW 837004009; Zorginstituut Nederland.

Conflict of interest M. van Barreveld, M.G.W. Dijkgraaf, M. Hulleman, L.V.A. Boersma, P.P.H.M. Delnoy, M. Meine, A.E. Tuinenburg, D.A.M.J. Theuns, P.H. van der Voort, G.P. Kimman, E. Buskens, J.P.G. Tijssen, N. Bruinsma, T.E. Verstraelen, A.H. Zwinderman, P.H.F.M. van Dessel and A.A.M. Wilde declare that they have no competing interests.

Open Access This article is distributed under the terms of the Creative Commons Attribution 4.0 International License (http:// creativecommons.org/licenses/by/4.0/), which permits unrestricted use, distribution, and reproduction in any medium, provided you give appropriate credit to the original author(s) and the source, provide a link to the Creative Commons license, and indicate if changes were made.

\section{References}

1. Mirowski M, Reid PR, Mower MM, et al. Termination of malignant ventricular arrhythmias with an implanted automatic defibrillator in human beings. N Engl J Med. 1980;303:322-4.

2. The Antiarrhythmics versus Implantable Defibrillators (AVID) Investigators. A comparison of antiarrhythmic-drug therapy with implantable defibrillators in patients resuscitated from near-fatal ventricular arrhythmias. N Engl J Med. 1997;337:1576-83.

3. Bardy GH, Lee KL, Mark DB, et al. Amiodarone or an implantable cardioverter-defibrillator for congestive heart failure. N Engl J Med. 2005;352:225-37.

4. Connolly SJ, Gent M, Roberts RS, et al. Canadian implantable defibrillator study (CIDS): a randomized trial of the implantable cardioverter defibrillator against amiodarone. Circulation. 2000;101: 1297-302.

5. Kadish A, Dyer A, Daubert JP, et al. Prophylactic defibrillator implantation in patients with nonischemic dilated cardiomyopathy. N Engl J Med. 2004;350:2151-8.

6. Kuck KH, Cappato R, Siebels J, Ruppel R. Randomized comparison of antiarrhythmic drug therapy with implantable defibrillators in patients resuscitated from cardiac arrest: the Cardiac Arrest Study Hamburg (CASH). Circulation. 2000;102:748-54.

7. Moss AJ, Hall WJ, Cannom DS, et al. Improved survival with an implanted defibrillator in patients with coronary disease at high risk for ventricular arrhythmia. Multicenter Automatic Defibrillator Implantation Trial Investigators. N Engl J Med. 1996;335:1933-40.

8. Moss AJ, Zareba W, Hall WJ, et al. Prophylactic implantation of a defibrillator in patients with myocardial infarction and reduced ejection fraction. N Engl J Med. 2002;346:877-83.

9. McMurray JJ, Adamopoulos S, Anker SD, et al. ESC Guidelines for the diagnosis and treatment of acute and chronic heart failure 2012: The Task Force for the Diagnosis and Treatment of Acute and Chronic Heart Failure 2012 of the European Society of Cardiology. Developed in collaboration with the Heart Failure Association (HFA) of the ESC. Eur Heart J. 2012;33:1787-847.

10. Yancy CW, Jessup M, Bozkurt B, et al. 2013 ACCF/AHA guideline for the management of heart failure: a report of the American College of Cardiology Foundation/American Heart Association Task Force on Practice Guidelines. J Am Coll Cardiol. 2013;62:e147-239.

11. Koller MT, Schaer B, Wolbers M, et al. Death without prior appropriate implantable cardioverter-defibrillator therapy: a competing risk study. Circulation. 2008;117:1918-26.

12. Moss AJ, Greenberg H, Case RB, et al. Long-term clinical course of patients after termination of ventricular tachyarrhythmia by an implanted defibrillator. Circulation. 2004;110:3760-5.

13. van Rees JB, Borleffs CJ, van Welsenes GH, et al. Clinical prediction model for death prior to appropriate therapy in primary prevention implantable cardioverter defibrillator patients with ischaemic heart disease: the FADES risk score. Heart. 2012;98:872-7.

14. Brignole M. Are complications of implantable defibrillators underestimated and benefits over-estimated? Europace. 2009;11:1129-33.

15. Poole JE, Johnson GW, Hellkamp AS, et al. Prognostic importance of defibrillator shocks in patients with heart failure. N Engl J Med. 2008;359:1009-17.

16. van Rees JB, Borleffs CJ, de Bie MK, et al. Inappropriate implantable cardioverter-defibrillator shocks: incidence, predictors, and impact on mortality. J Am Coll Cardiol. 2011;57:556-62. 
17. Groeneveld PW, Matta MA, Suh JJ, Heidenreich PA, Shea JA. Costs and quality-of-life effects of implantable cardioverter-defibrillators. Am J Cardiol. 2006;98:1409-15.

18. Bracke FA, Dekker LR, van der Voort PH, Meijer A. Primary prevention with the ICD in clinical practice: not as straightforward as the guidelines suggest? Neth Heart J. 2009;17:107-10.

19. Buxton AE, Lee KL, Hafley GE, et al. Limitations of ejection fraction for prediction of sudden death risk in patients with coronary artery disease: lessons from the MUSTT study. J Am Coll Cardiol. 2007;50:1150-7.

20. Disertori M, Quintarelli S, Mazzola S, et al. The need to modify patient selection to improve the benefits of implantable cardioverterdefibrillator for primary prevention of sudden death in non-ischaemic dilated cardiomyopathy. Europace. 2013;15:1693-701.

21. Stecker EC, Vickers C, Waltz J, et al. Population-based analysis of sudden cardiac death with and without left ventricular systolic dysfunction: two-year findings from the Oregon Sudden Unexpected Death Study. J Am Coll Cardiol. 2006;47:1161-6.

22. Tung R, Zimetbaum P, Josephson ME. A critical appraisal of implantable cardioverter-defibrillator therapy for the prevention of sudden cardiac death. J Am Coll Cardiol. 2008;52:1111-21. doi:10. 1016/j.jacc.2008.05.058.

23. Yap YG, Duong T, Bland JM, et al. Optimising the dichotomy limit for left ventricular ejection fraction in selecting patients for defibrillator therapy after myocardial infarction. Heart. 2007;93:832-6.

24. Comparative Effectiveness Research to Assess the Use of Primary ProphylacTic Implantable Cardioverter Defibrillators in Europe (EU-CERT-ICD) [cited 201420 August]. Available from: https:// clinicaltrials.gov/ct2/show/NCT02064192.

25. Efficacy of Implantable Cardioverter Defibrillator in Patients With Non-ischemic Systolic Heart Failure on Mortality (DANISH) [cited 201420 August]. Available from: https://clinicaltrials.gov/ ct2/show/NCT00541268.

26. Cowie MR, Marshall D, Drummond M, et al. Lifetime cost-effectiveness of prophylactic implantation of a cardioverter defibrillator in patients with reduced left ventricular systolic function: results of Markov modelling in a European population. Europace. 2009;11:716-26.

27. Mark DB, Nelson CL, Anstrom KJ, et al. Cost-effectiveness of defibrillator therapy or amiodarone in chronic stable heart failure: results from the Sudden Cardiac Death in Heart Failure Trial (SCDHeFT). Circulation. 2006;114:135-42.

28. Sanders GD, Hlatky MA, Owens DK. Cost-effectiveness of implantable cardioverter-defibrillators. N Engl J Med. 2005;353: 1471-80.
29. Sheldon R, O'Brien BJ, Blackhouse G, et al. Effect of clinical risk stratification on cost-effectiveness of the implantable cardioverterdefibrillator: the Canadian implantable defibrillator study. Circulation. 2001;104:1622-6.

30. Smith T, Jordaens L, Theuns DA, et al. The cost-effectiveness of primary prophylactic implantable defibrillator therapy in patients with ischaemic or non-ischaemic heart disease: a European analysis. Eur Heart J. 2013;34:211-9.

31. Zwanziger J, Hall WJ, Dick AW, et al. The cost effectiveness of implantable cardioverter-defibrillators: results from the Multicenter Automatic Defibrillator Implantation Trial (MADIT)-II. J Am Coll Cardiol. 2006;47:2310-8.

32. OpenClinica Open Source EDC n.d. Available from: https://www. openclinica.com/. Accessed 9 November 2016.

33. Goldenberg I, Vyas AK, Hall WJ, et al. Risk stratification for primary implantation of a cardioverter-defibrillator in patients with ischemic left ventricular dysfunction. J Am Coll Cardiol. 2008;51:288-96.

34. Al-Khatib SM, Hellkamp A, Curtis J, et al. Non-evidence-based ICD implantations in the United States. JAMA. 2011;305:43-9.

35. Lee DS, Birnie D, Cameron D, et al. Design and implementation of a population-based registry of Implantable Cardioverter Defibrillators (ICDs) in Ontario. Heart Rhythm. 2008;5:1250-6.

36. Krumholz HM. Registries and selectio bias. The need for accountability. Circ Cardiovasc Qual Outcomes. 2009;2(6):517-8.

37. Roovers JP. Registries: what level of evidence do they provide? Int Urogynecol J Pelvic Floor Dysfunct. 2007;18:1119-20.

38. Lee DS, Hardy J, Yee R, et al. Clinical risk stratification for primary prevention Implantable Cardioverter Defibrillators. Circ Heart Fail. 2015;8:927-37.

39. Sabbag A, Suleiman M, Laish-Farkash A, et al. Contemporary rates of appropriate shock therapy in patients who receive implantable device therapy in a real-world setting: From the Israeli ICD Registry. Heart Rhythm. 2015;12:2426-33.

40. Kober L, Thune JJ, Nielsen JC, et al. Defibrillator Implantation in Patients with Nonischemic Systolic Heart Failure. N Engl J Med. 2016;375:1221-30.

41. Goldenberg I, Gillespie J, Moss AJ, et al. Long-term benefit of primary prevention with an implantable cardioverter-defibrillator: an extended 8-year follow-up study of the Multicenter Automatic Defibrillator Implantation Trial II. Circulation. 2010;122:1265-71. 